\title{
Contemporary approach to odontogenic keratocyst: a review of the literature and case report
}

\begin{abstract}
The aim of this study was to evaluate the efficacy of the Odontogenic Keratocyst treatment through enucleation and cryotherapy with liquid nitrogen in a lesion that had previously been biopsied and also to compare the microscopic characteristic of the enucleated lesion to the biopsy microscopy. Enucleation was performed in a 27-year-old female patient with previously diagnosis by initial biopsy. The lesion extended from left mandibular body region to the ramus ipsilateral. The use of this technique proved to be an effective treatment. It is believed that the initial biopsies allowed a decrease in the size of the lesion and increase the thickness of the fibrous capsule, which prevent the rupture of the keratocyst capsule.
\end{abstract}

Keywords: keratocystic odontogenic, enucleation, cryoterapy

\author{
Volume II Issue 3 - 2020
}

\author{
Leonardo Matos Santolim Zanettini,' Maiara \\ Fumagalli, ${ }^{2}$ Claiton Heitz, ${ }^{3}$ Guilherme \\ Genehr Fritscher, ${ }^{3}$ Rogerio Miranda \\ Pagnoncelli ${ }^{3}$ \\ 'Department of Oral and Maxillofacial Surgery, University \\ Hospital Münster, Germany, Pontifical Catholic University of Rio \\ Grande do Sul, Brazil \\ ${ }^{2}$ Department of Pathology and Stomatology, Pontifical Catholic \\ University of Rio Grande do Sul, Brazil \\ ${ }^{3}$ Department of Oral and Maxillofacial Surgery, Pontifical \\ Catholic University of Rio Grande do Sul, Brazil
}

\begin{abstract}
Correspondence: Leonardo Matos Santolim Zanettini, Department of Oral and Maxillofacial Surgery, University Hospital Münster, Germany, Pontifical Catholic University of Rio Grande do Sul, Brazil, Tel 491522233303।,

Email leonardo.zanettini@acad.pucrs.br
\end{abstract}

Received: February 27, 2020 | Published: June 15, 2020

\section{Introduction}

The Odontogenic Keratocyst, ${ }^{1}$ first described by Philipsen in 195-61, was classified as a benign odontogenic tumor by the World Health Organization (WHO) in 2005, due to its aggressive nature and intrinsic growth potential, ${ }^{2}$ although some researchers believed that this pathology was more correctly considered a development cyst. $^{3}$ According to the latest WHO classification in 2017, the Odontogenic Keratocyst was moved from the neoplastic category to the cyst category, returning to its initial terminology and being listed as Odontogenic Keratocyst in the classification of odontogenic developmental cysts. This change was due the fact that many researchers suggest that the resolution of the cyst after marsupialization was not compatible with a neoplastic process. ${ }^{4}$

With odontogenic origin, from the remains of the dental lamina, the Odontogenic Keratocyst is a benign lesion, but potentially aggressive, and with an infiltrative behavior. ${ }^{1}$ It is histologically characterized by a cystic cavity, containing or not keratin, covered by a thin strip of squamous epithelium stratified keratinized and fibrous capsule. ${ }^{5}$ The epithelial lining has a corrugated appearance, with few cells in thickness, usually free of inflammation. The basal layer of the epithelium is composed of a palisade layer of epithelial cells, often hyperchromatic. Detachment of portions of the fibrous capsule epithelium can also be observed. Withthe presence of inflammatory changes, there may be changes in the typical histological characteristics of the lesion. ${ }^{6}$

It is more commonly found in patients between the second and third decades of life, its prevalence is slightly higher in men than in women and preferentially affects the mandible, with a tendency for involvement of the branch and angle regions. ${ }^{7}$ When located in the maxilla, these lesions are generally smaller than the mandibular ones. ${ }^{8}$
Odontogenic keratocysts are often asymptomatic and discovered in routine radiographic examinations. It only become clinically evident after bone expansion or secondary infection. When symptomatic, usually when it reach larger dimensions, signs ofpain, edema and drainage. However, there are cases of large injuries where there are no symptoms. ${ }^{1}$ Radiographically, it is shown as a unilocular radiolucent lesion, and may also be multilocular, with sclerotic margins well defined, and may be associated with an impacted tooth, most commonly the lower third molar, root resorption of the teeth involved and displace roots or causing extrusion of erupted teeth. ${ }^{7}$ The lesion can reach different dimensions and grow preferentially in the medullary cavity, in the anteroposterior direction, without causing considerable bone expansion. ${ }^{9}$ The rapid growth of an Odontogenic Keratocyst, when observed, may occur due to an active epithelial lining with a high proliferation rate. ${ }^{10}$ The differential diagnosis can be made comparing with dentigerous cyst, root cyst, lateral periodontal cyst and ameloblastoma. Radiographicaly, it is difficult to interpret and easy to be confused, histopathological analysis is necessary for the definitive diagnosis and definition of the treatment plan. Incisional and aspiration biopsies are the most used to obtain the sample. ${ }^{7}$ Relapses of this lesion is due to its cystic lining being fragile and thin, facilitating the permanence of remnants of the lesion after surgical removal and the difficulty of being totally removed. ${ }^{1}$ In addition to the fragility of the cystic lining, young people, multilocular lesions, large dimensions and greater length in the anteroposterior dimension, ${ }^{9}$ limited surgical accesses and extravasation of keratin in soft tissues are also considered risk factors for recurrence. ${ }^{5}$ Odontogenic keratocysts are usually the first manifestation of Basal Cell Nevoid Carcinoma Syndrome, also known as Gorlin's Syndrome, ${ }^{11}$ which is characterized by an inherited autosomal dominant syndrome that presents several clinical manifestations. The diagnosis of this syndrome can be made 
when two main criteria are present, or one main and two secondary criteria. The main criteria include basal cell carcinoma of the skin, odontogenic keratocyst, ectopic calcifications, vertebral abnormalities such as bifid ribs, skull calcification and family history. Minor criteria include skeletal anomalies, macrocephaly, protrusion of the frontal and parietal bone and cleft palat. $^{12}$

There are significant clinical and histological differences between solitary lesions and multiple lesions associated with Gorlin's syndrome. Multiple lesions are usually diagnosed in younger patients, more maxillary lesions are observed, the association, satellite cysts and proliferation of odontogenic epithelium in the connective tissue wall, when compared to solitary cases. ${ }^{13}$

The traditional conservative method for treatment is enucleation, however due to the high recurrence rate of these lesions, this method alone is not considered sufficient. Therefore, treatments such as cryotherapy, peripheral osteotomy (curettage), excision of the adherent mucosa, electrocoagulation, Carnoy's solution, marsupialization and decompression have been used. Some authors suggest that techniques such as marsupialization followed by enucleation are an even more conservative method and reduce treatment morbidity, as well as the chance of injury to adjacent vital structures. Others claim that more invasive techniques, such as resection, are more likely to use and are indicated for the most recurrent cases, due to the aggressiveness and morbidity of this technique. ${ }^{7}$ Conservative treatment has a higher risk of recurrence than more radical treatments, i.e, those that include extractions of all teeth involved with the injury and surgical resections. $^{11}$

Enucleation followed by curettage or osteotomy of the cystic cavity is performed with the aim of mechanically removing the remnants of the lesion and satellite microcysts from the bone walls, in order to reduce the recurrence rate. However, this method can affect adjacent structures such as the lower alveolar nerve region or increase existing fenestrations when the bone wall is perforated, exposing the overlapping mucosa, which may contain residual microcysts. ${ }^{9}$

Cryotherapy as a complementary procedure to enucleation has similar effects to curettage in relation to the elimination of residual satellite microcysts in the bone structure. In addition, the use of this solution after enucleation makes it possible to reach the soft tissues on the lesion, which may contain remnants, especially in regions of bone fenestrations that are difficult to access. Cryotherapy is a method that can be considered superior when compared to curettage or peripheral osteotomy as an adjuvant procedure. It has been reported that the recurrence may arise from remnants of the soft tissue, thus, it is indicated the removal of the mucosa on the lesion, mainly in the region of bone fenestrations. ${ }^{9}$

Decompression aim to reduce the pressure, while marsupialization is the creation of an opening that has the capacity to maintain itself, thus being a means of decompression. ${ }^{14}$ Some authors have described that the treatment with decompression facilitates enucleation, preventing the epithelial lining, which is attached to the underlying connective tissue, to break or separate the cystic wall during surgical removal of the lesion. These possible changes in biological behavior are likely consequences of inflammation in the region, through decompression opening, which also decreases intra-cystic pressure. ${ }^{3}$

The postoperative complications resulted from the treatment the most common is paresthesia, which may be caused during the removal of the lesion or cell damage caused by the use of cryotherapy. Mandibular fracture has also been reported due to low amount of remaining bone or weakening of the bone, resulting from the use of cryotherapy. ${ }^{9}$

The monitoring of patients should be carried out periodically. As the recurrence of these lesions can be detected in up to five years after surgery, ${ }^{2}$ a biannual reassessment during the first year is recommended. Thereafter the patient must be followed up every year, since recurrences may appear after a long period of time. The tomographic follow-up must be carefully performed, as it allows the diagnosis of recurrent lesions in the initial stages, ensuring a second surgical intervention with greater safety and less morbidity. ${ }^{15}$

The aim of this study was to present a case report of a young patient undergoing a surgical procedure for curettage of Odontogenic Keratocyst involving dental extractions in the mandible and additional local cryotherapy, and rehabilitation of the edentulous.

\section{Case report}

Female patient, 27 years old, attended the School of Dentistry of the Pontifical Catholic University of Rio Grande do Sul (FO-PUCRS), at the ambulatory of Oral and Maxillofacial Surgery with an extensive lesion in the mandible, of slow and non-painful growth on the left side, slightly increased in size, with approximately six months of evolution, compatible with Odontogenic Keratocyst (Figure 1). Previously, the patient had already performed an incisional biopsy of the lesion, and the histopathological diagnosis was confirmed, being compatible with the clinical and radiographic diagnosis of the lesion. The biopsy was also evaluated by the FO-PUCRS pathology service (Figure 2). Clinical examination showed a slight increase in extra-oral volume in the region of the left mandibular angle and the presence of intra-oral fistula in the region of the lower premolars, suggesting an involuntary marsupialization. The tomographic image showed a radiolucent, unilocular lesion, of anteroposterior growth in the medullary bone, in the region of the body and mandibular branch, without causing considerable bone expansion, measuring approximately $5 \mathrm{~cm}$ in length and $2 \mathrm{~cm}$ in height with some bone fenestrations, buccal and lingual (Figures $3 \& 4$ ). Considering the patient's age, the extent of the lesion, the presence of non-healing oral fistula, which suggested an involuntary marsupialization and the patient's desire for a more conservative treatment option, enucleation followed by curettage and cryotherapy as an adjuvant treatment was planned to decrease the chances of recurrence and, in case of mandibular fracture, installation of a reconstruction plate. The patient was referred to the Orthodontics Clinic of FO-PUCRS, for the manufacture of a fixed appliance for maxillo-mandibular block after surgery. A prototype of the mandible was performed (Figure 5) in order to better access the extent of the lesion and for surgical planning in case of need to use a reconstruction plate.The surgical procedure was performed under general anesthesia and nasal intubation. An intra-sulcular incision was made from the retromolar region to the first premolar and mucoperiosteal flap. Access to the lesion was obtained through the creation of a bone window. The extraction of teeth 37, 36 and 35 was necessary. Enucleation and complete removal of the lesion was performed, followed by curettage and excision of the overlying mucosa and periosteum that were in contact with the lesion due to bone fenestrations (Figure 6). The adjacent soft tissues were protected with gauze and nitrogen was applied for cryotherapy in the surgical bed. The suture was performed with vicryl 4-0 and the maxillo-mandibular block was immediate due to the fragility of the mandible (Figure 7). The collected material was sent for histopathological analysis at the FO-PUCRS Pathology Service, to compare the current histological characteristics, in relation to those of the primary slide (Figure 8). 


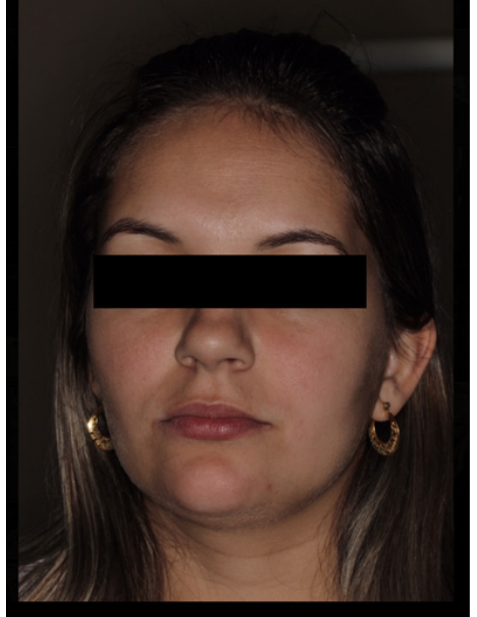

Figure I Initial photograph. Clinical aspect of a slight increase in volume in the left-side mandibular angle region.

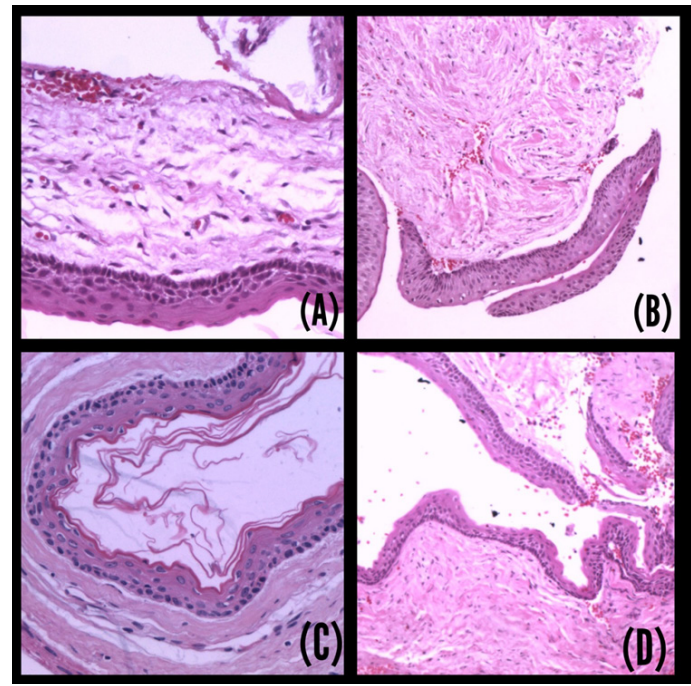

Figure 2 Initial microscopy. (A) Epithelial lining with a thickness of 6 to 8 cells, with a layer of hyperchromatic basal cells and palisade. (B) Epithelium standing out from the conjunctive. (C) Corrugated paraceratinized surface, presence of keratin. (D) General microscopic appearance.

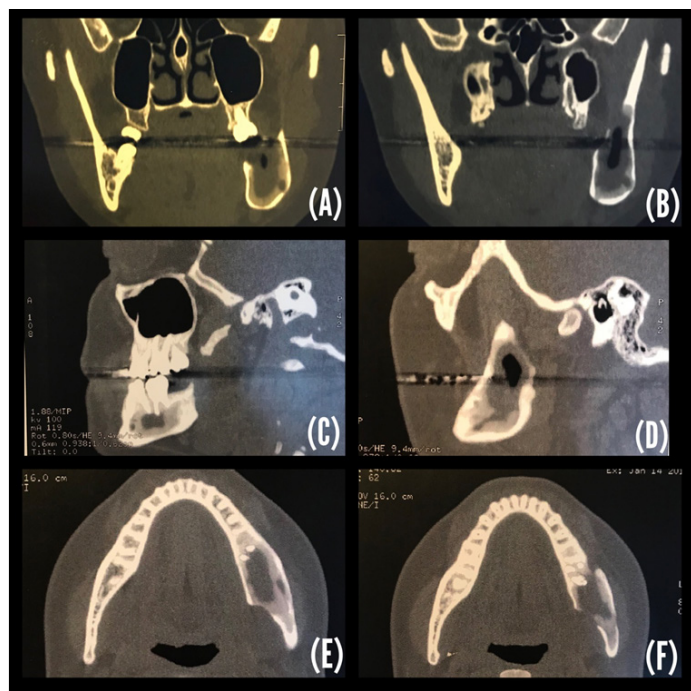

Figure 3 Preoperative computed tomography image. (A, B) Ccoronal sections. (C, D) Sagittal cuts. (E, F) Axial cuts.

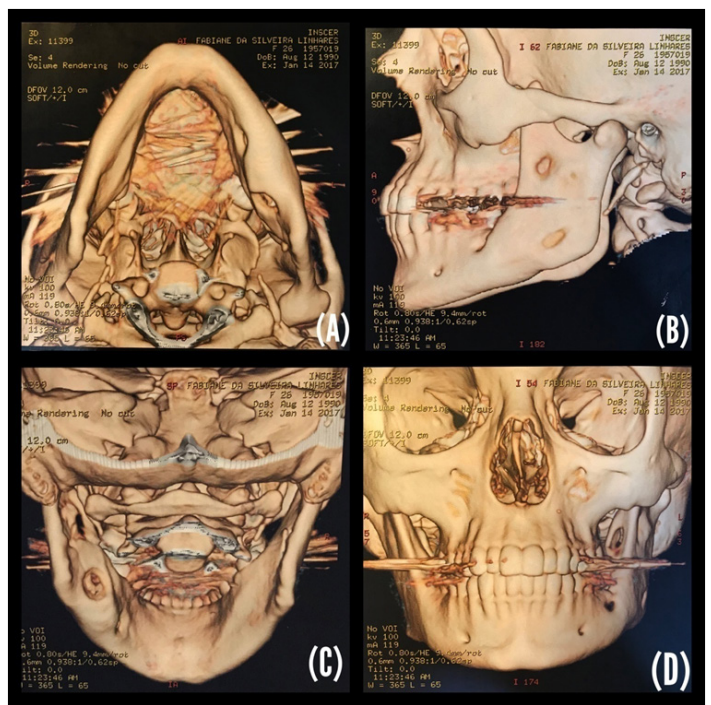

Figure 4 3D reconstruction of computed tomography. (A) Axial view. (B) Side view. (C) Postero-anterior view;. (D) Antero-posterior view.

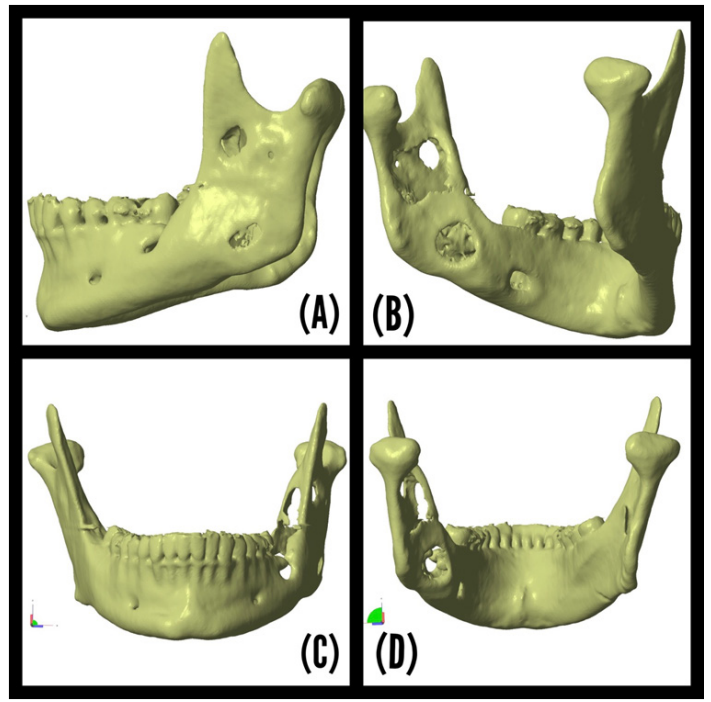

Figure 5 Mandible prototyping. (A) side view. (B) Lateral-anterior-posterior view. (C) Anteroposterior view. (D) Poster-anterior view.

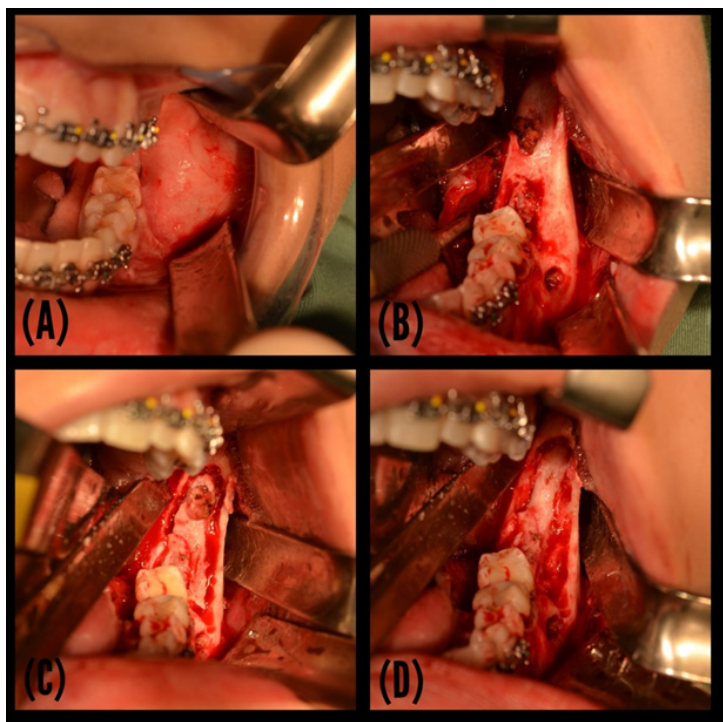

Citation: Zanettini LMS, Fumagalli M, Heitz C, et al. Contemporary approach to odontogenic keratocyst: a review of the literature and case report. J Dent Health Oral Disord Ther. 2020; I I (3):7 I-76. DOI: I0.I5406/jdhodt.2020.I I.00522 


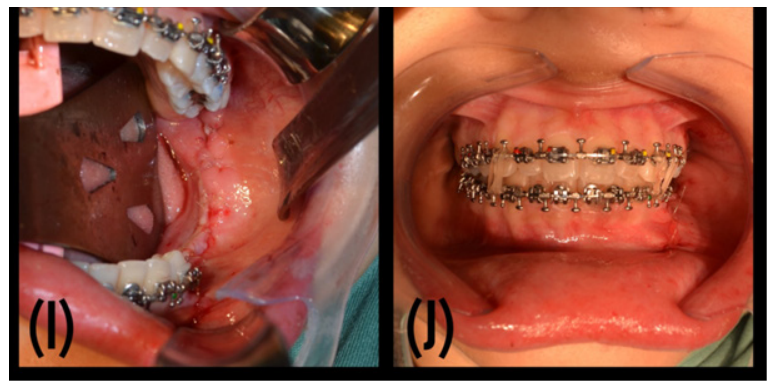

Figure 6 Surgical procedure. (A) Preoperative. (B) Surgical access-presence of bone fenestrations. (C) Peripheral osteotomy to access the lesion. (D) Surgical exposure of the lesion to enucleation. (E) Complete removal of the lesion. (F) Teeth 37,36 and 35 that were involved in the lesion. $(\mathrm{G}, \mathrm{H})$ Bone cavity after enucleation and curettage and prior to cryotherapy. (I) Intraoral sutures. (J) Immediate postoperative period.

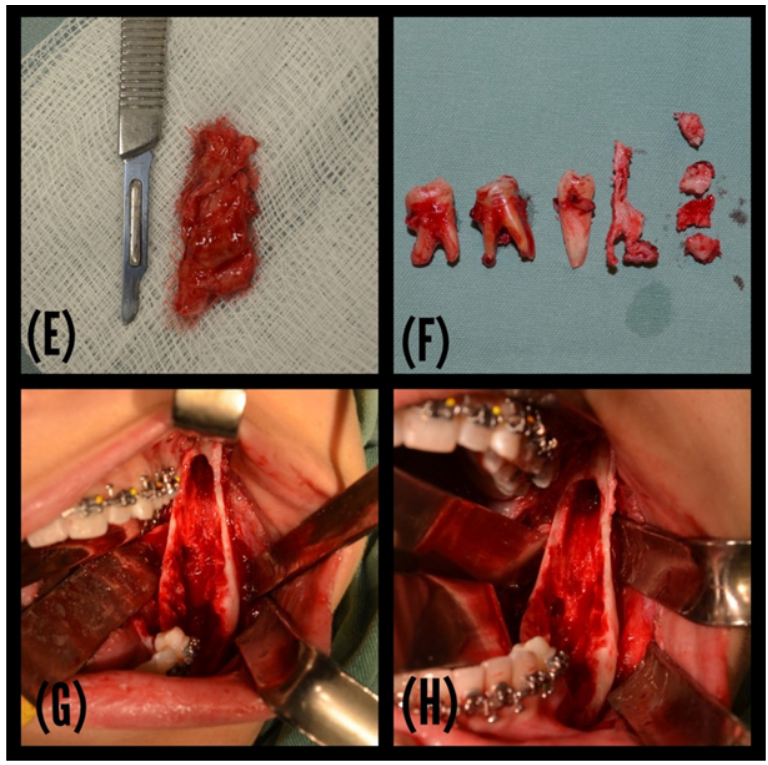

Figure 7 Maxillo-mandibular block.

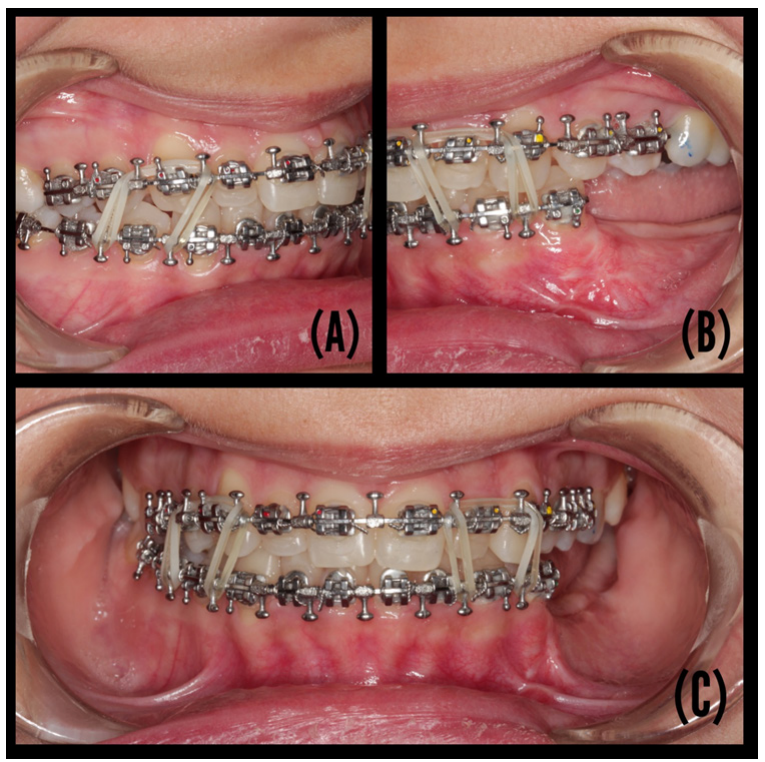

Figure 8 Final microscopy. (A, B) increasing the thickness of the epithelial lining. (C) Epithelium standing out from the conjunctive.(D) slightly corrugated epithelial surface.
The result of the histopathological examination confirmed the diagnosis of Odontogenic Keratocyst, and since this lesion is highly recurrent, the patient was informed of the need for regular follow-up.

On the seventh postoperative day, the patient returned with edema in the operated region, due to the surgical procedure, absence of painful symptoms and healing of the surgical wound without complications.

After 15 days postoperatively, a significant reduction in edema was observed. Panoramic radiography was requested, which showed a good remaining mandibular bone structure, which made it possible to remove the maxillo-mandibular block. The patient continued under food restriction guidance due to bone fragility and limited mouth opening.

With 30 days postoperatively, the patient already had completed healing of the surgical wound, complete remission of the edema and still slight limitation of mouth opening. The patient was followed up, a radiographic control was performed with panoramic radiographs every 2 months.

After the 6-month postoperative period, the patient underwent a complete mandible tomography in which significant bone neoformation can be observed without areas suggestive of lesion recurrence (Figure 9).

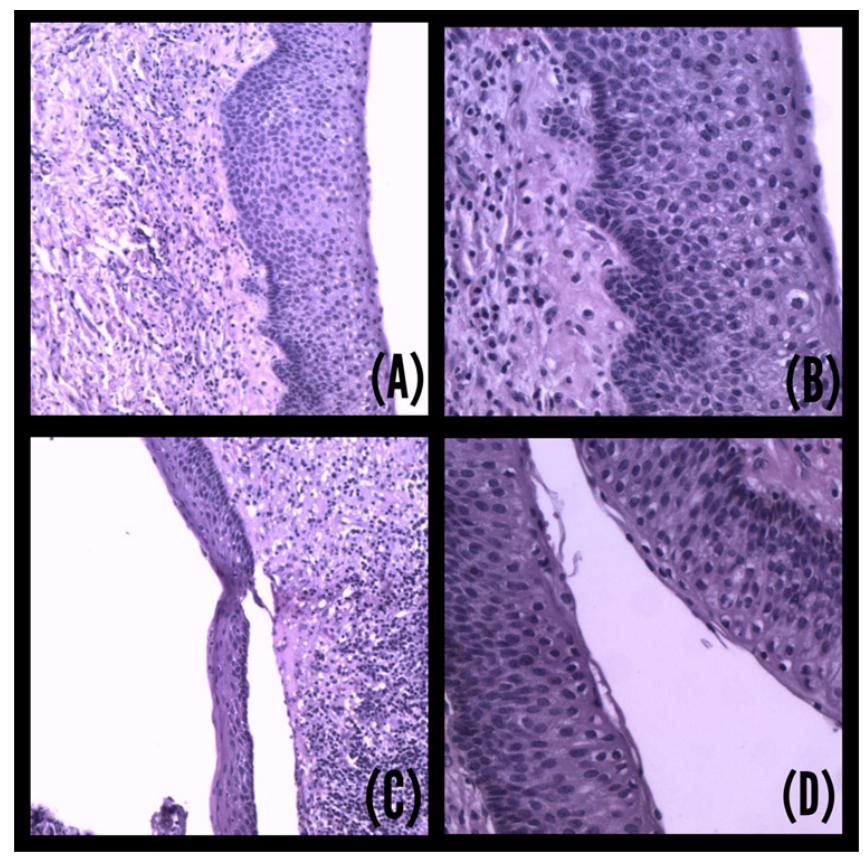

Figure 9 6-month postoperative tomographic image.

Considering the good evolution of the clinical picture, the patient's desire and age, prosthetic rehabilitation with osseointegrated implants was then planned. Surgical planning consisted of molding and scanning the study models, virtual planning in digital software with 3D surgical guide printing, placement of 2 Straumann SLA $4.1 \mathrm{~mm}$ $\mathrm{x} 10 \mathrm{~mm}$ Roxolid implants and bone graft with biomaterial (Bio Oss $0.5 \mathrm{~g})$. In the surgical plan, there was not enough mouth opening for surgical milling. The guide was fixed and only the initial reference perforations were made for the surgery segment. The implants were installed with good stability and the vestibular region of the implants was filled with biomaterial. Prosthetic healers were placed over the implants (Figure 10). At 7 days postoperatively, the surgical wound was well healed and had no signs of infection. 


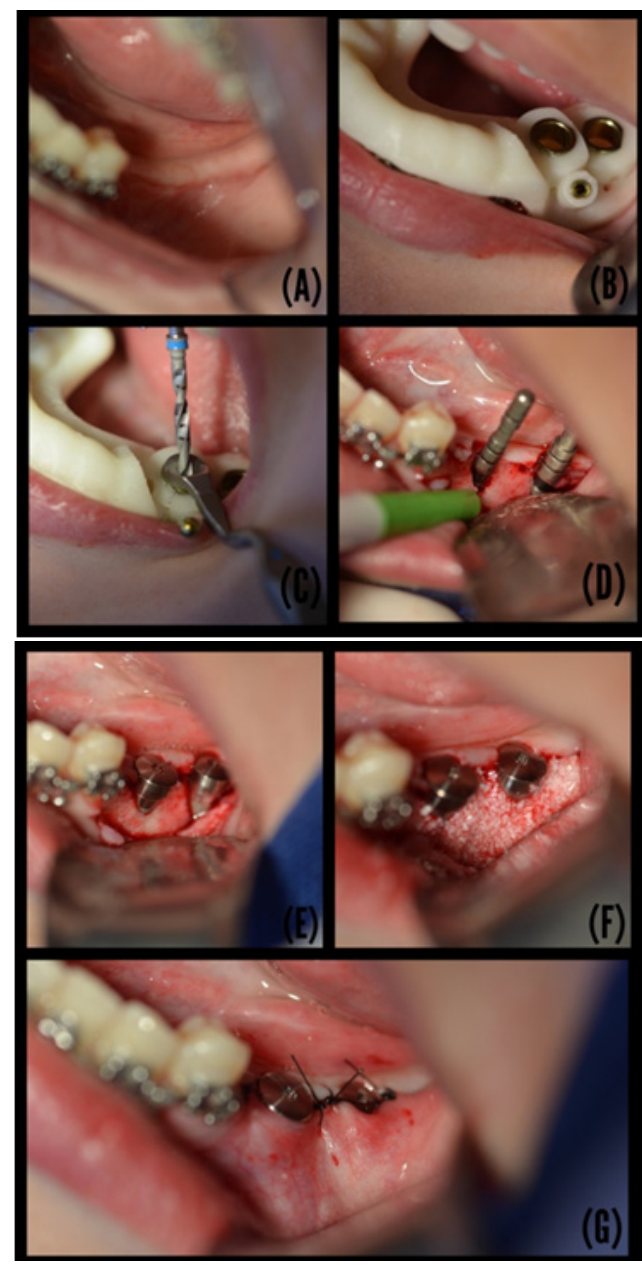

Figure 10 Surgical procedure for implant placement. (A) Preoperative. (B) Installation of the prototyped surgical guide. (C) Initial milling for reference. (D) Direction indicators in position. (E) Implants installed with prosthetic healers. (F) Biomaterial bone graft in the buccal wall. (G) Intraoral sutures.

\section{Discussion}

The odontogenic keratocyst is a cyst of uno or multilocular intraosseous development, with a stratified paraceratinized squamous epithelium, with aggressive local infiltrative behavior and high recurrence rates. ${ }^{16}$ They are generally asymptomatic and have a considerable predilection for the the mandibular region, causing no significant bone expansion. ${ }^{17}$ In the presented case, the lesion was asymptomatic, located in the body and left mandibular branch, with growth in the anteroposterior direction causing a slight increase in extra oral volume.

Histologically, this lesion shows a cystic cavity containing or not keratin, covered by a few layers of cells of corrugated paraceratinized squamous epithelium, with the cells of the basal layer arranged in a palisade pattern and little thickness of the fibrous capsule. Often, the epithelium is detached from the conective tissue. ${ }^{18}$ The lesion in this study revealed classic histopathological characteristics of an odontogenic keratocyst, standing out from the epithelium in some regions and with the presence of keratin, which can be easily diagnosed.

Among the different treatment methods cited in the literature, there is none that is associated with a zero recurrence rate. Surgical techniques and treatment modalities has been described over the years and can vary between more radical to conservative techniques. According to Al-Moraiissi et al., ${ }^{19}$ 1marsupialization and decompression as the definitive treatment is the method with the highest rate of recurrence, followed by simple enucleation and peripheral osteotomy, respectively. Resections have a lower recurrence rate, followed by enucleation with application of Carnoy's solution, enucleation with application of cryotherapy with liquid nitrogen and decompression with subsequent residual cystectomy, respectively. ${ }^{19}$ The choice in the presetend case was a more conservative treatment in relation to what had been proposed, regarding the patient's age and desire for a less invasive technique. The technique of enucleation and application of cryotherapy with liquid nitrogen was used; as there was no mandibular fracture during the removal of the lesion, maxillomandibular block was performed as an alternative to placing a reconstruction plate to prevent bone fracture. It has been reported that relapse can arise from residual microcysts on the overlapping mucosa, if not removed, especially when there are bone fenestrations. The use of cryotherapy as an adjunctive procedure to enucleation can be effective in removing these remaining cells. ${ }^{9}$ Cryotherapy uses low temperature to induce tissue necrosis and maintains bone architecture, unlike Carnoy's solution, which destroys osteogenic and osteoconductive properties. Liquid nitrogen is the most efficient cryogenic agent and can achieve a deep freezing effect. The necrosis of cells and tissues by direct cell injury is due to an osmotic and electrolyte imbalance and vascular changes that occur after the thawing period. Despite the benefits of this technique, bone tissue becomes fragile and prone to pathological fracture. ${ }^{17}$

Marsupialization involves the formation of a cavity that remains open to the oral environment, reducing intra-cystic pressure, allowing the lesion to slowly decrease in size and increase the thickness of the fibrous capsule, facilitating posterior enucleation and preserving vital structures when involved by the lesion. ${ }^{16}$ As observed in this case, there was an involuntary marsupialization due to the absence of healing in the biopsied region, maintaining communication of the lesion with the oral environment. During enucleation, an increase in the fibrous capsule can be seen, which facilitated the complete removal of the lesion. The histological findings of the sample sent for analysis confirmed the that there was a phenomenon similar to marsupialization, due to the alteration of the lesion's characteristics when compared to the initial slide; probable result of the local inflammatory process that was generated by the maintenance of the open cavity, with constant contamination. Microscopically, there was an increase in the thickness of the capsule and epithelium and the presence of an inflammatory infiltrate, which could be suggestive of another odontogenic cyst, if there was no previous histopathological diagnosis. Some classic characteristics of keratocyst remained, such as para-keratinization, the presence of corrugated epithelium and cells of the basal layer in palisades in some regions, few desquamative cells and the detachment of the conjunctival epithelium.

Although the Odontogenic Keratocyst is widely known for its aggressive nature and high relapse capacity, ${ }^{20}$ it was chosen together with the patient, considering the benefits and possible complications, for an early rehabilitation, due to the good prognosis of the clinical condition and the age of the patient.

\section{Conclusion}

The use of the enucleation technique followed by cryotherapy with liquid nitrogen and subsequent maxillomandibular block was an effective and relatively conservative approach, when compared to 
local resection and placement of a mandibular reconstruction plate, for the treatment of odontogenic keratocyst. The use of this technique allowed the preservation of the adjacent anatomical structures and allowed a decrease in the size of the lesion and an increase in the thickness of the fibrous capsule, which facilitated its enucleation, which is characteristic of the marsupialization technique. To achieve the lowest morbidity, the best prognosis and the maintenance of the patient's quality of life, resections should be reserved for recurrent cases, multiple or syndromic lesions and, as a less aggressive alternative, enucleation with the application of cryotherapy is shown as an effective treatment with low recurrence rates.

\section{Funding}

None.

\section{Acknowledgments}

None.

\section{Conflicts of interest}

The authors have no conflicts of interest to declare.

\section{References}

1. Sharif FNJ, Oliver R, Sweet C, et al. Interventions for the treatment of keratocystic odontogenic tumours. Cochrane Database of Systematic Reviews. 2015.

2. Kishor Gupta R, Govind Dugal A, Ramlal Pawar S, et al. A Rare Simultaneous Occurrence of Odontogenic Keratocyst and Unicystic Ameloblastoma in Mandible: A Case Report. J Clin Diagn Res. 2016;10(8):ZD01-ZD04.

3. Sarah Awni, Conn B. Oral Surgery, Decompression of keratocystic odontogenic tumors leadingto increased fibrosis, but without any change in epithelial proliferation. J Oral Maxillofac Surg. 2017;123(6):634-644.

4. Soluk-Tekkesin M, Wright JM. The world health organization classification of odontogenic lesions: a summary of the changes of the $20174^{\text {th }}$ edition. Turk J Pathol. 2018;34(1).

5. Alstad V, Abtahi J. Surgical removal of keratocystic odontogenic tumours via a Le Fort I osteotomy approach: a retrospective study of the recurrence rate. Int J Oral Maxillofac Surg. 2017;46(4):434-439.

6. Neville BW, Damm DD, Allen CM, et al. Oral and Maxillofacial Pathology. 4 ed. Rio de Janeiro: Elsevier; 2016.

7. Díaz-Belenguer Á, Sánchez-Torres A, Gay-Escoda C. Role of carnoy’s solution in the treatment of keratocystic odontogenic tumor: A systematic review. Med Oral Patol Oral Cir Bucal. 2016;1;21(6):e689-e695.
8. Mortazavi H, Baharvand M. Jaw lesions associated with impacted tooth: A radiographic diagnostic guide. Imaging Sci Dent. 2016;46:147-157.

9. Leung YY, Lau SL, Tsoi KY, et al. Results of the treatment of keratocystic odontogenic tumours using enucleation and treatment of the residual bony defect with Carnoy's solution. Int J Oral Maxillofac Surg. 2016;45(9):1154-1158

10. Tobias L Pittl, Marius Meier, Paul Hakl, et al. Long-term observation of a large keratocystic odontogenic tumourofthe mandible treated by a single enucleation procedure: A case reportand literature review. Int J Surg. 2017;34:119-122.

11. Shimada Y, Maruoka Y, Yamaji I, et al. Non-Syndromic Familial Keratocystic Odontogenic Tumour: A Rare Case Report in Japanese Identical Twins. J Clin Diagn Res. 2016;10(8):ZD28-ZD30.

12. Dong-UkSeo, Su-Gwan Kim, Ji-Su Oh, et al. Treatment of nevoid basal cell carcinoma syndrome: a case report. J Korean Assoc Oral Maxillofac Surg. 2017;42(5):284-287.

13. Bello IO. Keratocystic odontogenic tumor: A biopsy service's experience with 104 solitary, multiple and recurrent lesions. Med Oral Patol Oral Cir Bucal. 2016; 1;21(5):e538-e546.

14. Park SY, Shin YJ, Kim CH, et al. Reconstruction of extensive jaw defects induced by keratocystic odontogenic tumor via patient-customized devices. Maxill of Plast Reconstr Sur. 2015;37:37.

15. Cunha JF,Gomes CC, de Mesquita RA, et al. Clinicopathologic features associated with recurrence of the odontogenic keratocyst: a cohort retrospective analysis. J Oral Maxillofac Pathol. 2016;121(6):629-635.

16. De Molon RS, Verzola MH, Pires LC, et al. Five years follow-up of a keratocyst odontogenic tumor treated by marsupialization and enucleation: A case report and literature review. Contemp Clin Dent. 2015;6(1):S106S110.

17. De Souza Cruz EL, da Silva Tabosa AK, Falcão AS, et al. Use of refrigerant spray of a propane/butane/isobutane gas mixture in the management of keratocystic odontogenic tumors: a preliminary study. Oral Maxillofac Surg. 2017;21(1):21-26.

18. Vázquez-Romero MC, Serrera-Figallo MA, Alberdi-Navarro J, et al. Maxillary peripheral keratocystic odontogenic tumor. A clinical case report. J ClinExp Dent. 2017;9(1):e167-e171.

19. Al-Moraissi EA, Dahan AA, Alwadeai MS, et al. What surgical treatment has the lowest recurrence rate following the management of keratocystic odontogenic tumor? A large systematic review and meta-analysis. J Craniomaxillofac Surg. 2017;45(1):131-144.

20. Kinard BE, Chuang SK, August M, et al. For treatment of odontogenic keratocysts,Is Enucleation, when compared to decompression, a less complex management protocol. J Oral Maxillofac Surg. 2015;73(4):641-648. 